\title{
Relationship Between Stereopsis and Vision-Related Quality of Life Following Intravitreal Ranibizumab Injections for Central Retinal Vein Occlusion
}

Fumiki Okamoto ( $\square$ fumiki-o@md.tsukuba.ac.jp )

University of Tsukuba

Mizuki Tomioka

University of Tsukuba

Tomoya Murakami

University of Tsukuba

Shohei Morikawa

University of Tsukuba

Yoshimi Sugiura

University of Tsukuba

Takahiro Hiraoka

University of Tsukuba

Tetsuro Oshika

University of Tsukuba

\section{Research Article}

Keywords: VR-QOL, central retinal vein occlusion (CRVO), Stereopsis, IVR

Posted Date: July 20th, 2021

DOl: https://doi.org/10.21203/rs.3.rs-724591/v1

License: (9) This work is licensed under a Creative Commons Attribution 4.0 International License.

Read Full License 


\section{Abstract}

The study aimed to evaluate changes in stereopsis and vision-related quality of life (VR-QOL) in patients with central retinal vein occlusion (CRVO) following intravitreal ranibizumab injection (IVR) and investigate the relationship between stereopsis and VR-QOL. This study included 23 treatment-naïve patients with CRVO and 13 age-matched normal controls. Stereopsis, best-corrected visual acuity (BCVA), VR-QOL, and retinal microstructures were examined pre-treatment and 12 months post-treatment. The Titmus Stereo Test (TST) and TNO stereotest (TNO) were used to evaluate stereopsis. VR-QOL was evaluated using the 25-item National Eye Institute Visual Function Questionnaire (VFQ-25). IVR immediately and significantly improved the TST values, TNO values, composite VFQ-25 score, BCVA, and central foveal thickness in patients with CRVO. The 12-month post-treatment TST and TNO values were significantly worse in the CRVO group compared to those in the normal group. The baseline composite VFQ-25 score significantly correlated with the TST value. Multivariate analysis revealed significant associations between the 12-month post-treatment composite VFQ-25 score and the baseline and 12month post-treatment TNO values. In conclusion, IVR immediately improved stereopsis in CRVO, albeit below normal levels. Stereopsis (not visual acuity) was associated with pre- and post-treatment VR-QOL in patients with CRVO.

\section{Introduction}

Stereopsis, which is one of the most advanced visual functions, is the ability to perceive the depth of the field through the parallax of images formed by both eyes. Strabismus is among the typical ocular disorders in which stereopsis is compromised owing to impaired coordination between both eyes. On the other hand, stereopsis can also be impaired owing to visual dysfunction in one eye. The most common parameter of visual function that affects stereopsis is visual acuity. Previous experimental studies have shown that blurred vision in one eye affects stereopsis [1-5]. Several studies have reported the impairment of stereopsis in unilateral retinal diseases such as retinal detachment (RD) [6,7] epiretinal membrane (ERM) [8,9], macular hole (MH) [10,11], and branch retinal vein occlusion (BRVO) [12]. Moreover, the stereopsis of patients with these conditions were worse than those of normal participants even following successful treatment $[6,9,11,12]$. However, no study has investigated stereopsis in patients with central retinal vein occlusion (CRVO).

The traditional ophthalmologic measures of clinical outcome, such as visual acuity, are increasingly being complemented by assessments of patients' visual function and perceived quality of life (QOL). The 25-item National Eye Institute Visual Function Questionnaire (VFQ-25) is a vision-related QOL (VR-QOL) instrument designed to assess patients' perception of their visual function and QOL [13]. The VFQ-25 has been used to track the outcomes of several retinal diseases including BRVO [14,15], ERM [9,16], RD [1719], $\mathrm{MH}$ [20], proliferative diabetic retinopathy (PDR) [21], and diabetic macular edema (DME) [21]. The CRUISE [22] and GALILEO studies [23] used the VFQ-25 to assess the VR-QOL in patients with CRVO and investigated the changes in the QOL following treatment. The deterioration in the VR-QOL in patients with retinal diseases is attributed to the impairment of visual acuity and various factors related to visual 
function. Metamorphopsia has been demonstrated to be the cause of decreased VR-QOL in patients with ERM $[16,21]$ and $\mathrm{MH}[20]$. The deterioration in contrast sensitivity has been demonstrated to be the cause of decreased VR-QOL in PDR [12], DME [21], after RD [17] and vitreous floaters [24]. Moreover, another

study reported that stereopsis following RD affects the patients' VR-QOL, especially while driving [19]. The present study aimed to assess the stereopsis and VR-QOL in patients with CRVO before and after treatment, and evaluate the relationship between stereopsis and VR-QOL.

\section{Results}

Table 1 presents the baseline characteristics of the patients with CRVO and normal controls. This study enrolled 23 patients with CRVO and 13 normal controls. The stereopsis (TST and TNO) and composite VFQ-25 scores were significantly worse in CRVO compared to normal controls. No differences were observed between the age or sex of the participants of the CRVO and normal groups. No patient with CRVO discontinued treatment during the study period. The mean number of injections during the treatment period was $5.6 \pm 2.0$ (range 3-8 injections). None of the patients experienced ocular treatmentemergent serious adverse events such as glaucoma, iris neovascularization, RD, vitreous hemorrhage, and endophthalmitis. None of the patients underwent cataract surgery in the affected eye or in the other eye during the follow-up period.

\section{Changes in stereopsis, VR-QOL, visual acuity, and CFT in the CRVO group}

Figure 1 depicts changes in the stereopsis and VR-QOL. The TST values of patients with CRVO at baseline and at $3,6,9$, and 12 months following treatment were $3.17 \pm 0.70,2.68 \pm 0.84,2.68 \pm 0.79$, $2.54 \pm 0.77$, and $2.73 \pm 0.78$, respectively. The TNO values of patients with CRVO at baseline and at 3,6 , 9 , and 12 months following treatment were $3.27 \pm 0.59,2.88 \pm 0.72,2.93 \pm 0.68,2.78 \pm 0.82$, and $2.79 \pm$ 0.76 , respectively. Significant improvements were observed in the TST and TNO values from 1 month following IVR injection (Figure 1A, B). The composite VFQ-25 scores at baseline and at 3, 6, and 12 months following treatment were $62.6 \pm 16.9,70.6 \pm 15.6,72.2 \pm 15.8$, and $74.3 \pm 14.6$, respectively. The composite VFQ-25 scores at 3,6 , and 12 months following treatment showed significant improvement compared to those at baseline ( $p<0.005, p<0.005$, and $p<0.005$, respectively) (Figure 2$)$.

Figure 2 depicts the changes in the BCVA and CFT. The BCVA at baseline and at 3, 6, 9, and 12 months following treatment was $0.79 \pm 0.56,0.43 \pm 0.49,0.51 \pm 0.51,0.55 \pm 0.57$, and $0.59 \pm 0.61 \log M A R$, respectively. Significant improvements were observed in the BCVA from 1 month after treatment (Figure $3 A$ ). The CFT at baseline and at 3, 6, 9, and 12 months following treatment was $770 \pm 319,181 \pm 58,361$ $\pm 303,375 \pm 308$, and $308 \pm 259 \mu \mathrm{m}$, respectively. Significant improvements were also observed in the CFT from 1 month following treatment (Figure 3B).

\section{Comparison of stereopsis between CRVO and normal controls}

The TST and TNO values of the normal controls were $1.72 \pm 0.19$ and $1.85 \pm 0.23$, respectively. These values in CRVO before and after treatment were worse than those of the normal controls (Figure 4A, B). 


\section{Relationship between VR-QOL and visual functions}

The composite VFQ-25 score at baseline significantly correlated with the TST values at baseline, while it was not associated with the TNO and BCVA values (Table 2). The composite VFQ-25 score showed a significant correlation with the TNO values and the BCVA 12 months post-treatment (Table 2).

Multivariate analysis revealed that the composite VFQ-25 score was significantly associated with the TNO values 12 months post-treatment $(p=0.019)$. The composite VFQ- 25 score 12 months posttreatment significantly correlated with the TST, TNO, and BCVA values at baseline (Table 2). Multivariate analysis revealed that the composite VFQ-25 score obtained 12 months posttreatment was significantly associated with the TNO values at baseline $(p=0.011)$.

\section{Discussion}

This study found that patients with CRVO had impaired stereopsis, which was worse than that in the normal controls. The baseline stereopsis $(\mathrm{log})$ in the patients with CRVO in this study was 3.17 as measured by the TST and 3.27 as measured by the TNO. This study was the first to investigate stereopsis in patients with CRVO. The impairment in the visual function of one eye is known to compromise stereopsis. Prior studies have investigated the disturbance of stereopsis in patients with unilateral retinal diseases, including RD [6,7], ERM [8,9], MH [10,11], and BRVO [12]. The stereopsis in patients with ERM was 2.35 on the TST and 2.84 on the TNO [9], 2.7 on the TST and 2.8 on the TNO in patients with $\mathrm{MH}$ [11], and 2.72 on the TST and 2.72 on the TNO in patients with BRVO [12]. IVR immediately improved stereopsis in patients with CRVO; however, it could not restore it to the level of normal controls. The stereopsis was 2.73 on the TST and 2.79 on the TNO 12 months post-treatment in patients with CRVO in this study. The stereopsis following treatment for ERM was 2.19 on the TST and 2.63 on the TNO [9], 2.2 on the TST and 2.4 on the TNO in MH [11], and 2.06 on the TST and 2.32 on the TNO in BRVO [12]. Therefore, the degree of stereopsis impairment in CRVO is worse than that in other retinal diseases both before and after treatment.

The BCVA and CFT improved immediately following treatment in patients with CRVO and remained stable throughout the subsequent 12-month period. The 12-month improvement in the BCVA was 0.2 logMAR (10 letters) in this study. The 12-month BCVA following treatment was 13.9 and 16.9 letters better than that of the baseline in the CRUISE [22] and GALILEO studies [23], respectively. The reason for the comparatively lower improvement in the BCVA in our study than that in the other studies could be attributed to the inclusion criteria. The BCVA for eligibility in the CRUISE [22] and GALILEO studies [23] were 20/40 to 20/320. On the other hand, our study did not set any BCVA value as an eligibility criterion, and thus, the BCVA in our study population ranged from $20 / 20$ to $20 / 2000$. The improvement in visual acuity could be comparatively lesser than that in the previous studies because our study included patients with extremely poor vision and good vision.

The composite VFQ-25 score improved from 62.6 points before treatment to 74.3 points 12 months following treatment. The mean increase in the composite VFQ-25 score (from baseline) was 9.6 points at 
6 months, and 11.7 points at 12 months following treatment in this study. The mean increase from the baseline VFQ-25 composite score was 7.1 points every 6 months following treatment, and 7.8 and 7.1 points every 12 months following treatment, respectively, in the GALILEO [23] and CRUISE studies [22]. On the other hand, the mean number of injections during the 12-month period in our study was 5.6, which was lower than that in the CRUISE (9.8) and GALILEO (11.8) studies. Despite these results, the composite VFQ-25 score in our study improved as much or more than that in other studies, suggesting that $3+P R N$ is acceptable as the standard treatment for CRVO from the perspective of the QOL.

The baseline composite VFQ-25 score was associated with the TST values, and not the BCVA. The composite VFQ-25 score was not related to the BCVA, but to the TNO values 12 months post-treatment. Moreover, multiple regression analysis determined the TNO value as a prognostic factor for the VR-QOL in patients with CRVO in this study. The relationship between visual acuity and VR-QOL is well known in diseases such as diabetic retinopathy [25] and uveitis [26]. In addition, the relationship between visual functions other than visual acuity and VR-QOL has been reported in several retinal diseases. Previous studies reported that VR-QOL was affected by metamorphopsia in patients with ERM [16,21] and MH [20] and that the VR-QOL was associated with contrast sensitivity in PDR [21] DME [21], after RD [17], and vitreous floaters [24]. Moreover, stereopsis was associated with the VR-QOL, especially during driving, following RD surgery [19]. Our results suggest that stereopsis is an important factor affecting the QOL of patients with CRVO.

The limitations of this study include its small sample size and short follow-up duration. We evaluated the patients for 12 months following treatment. In the COPERNICUS study, the BCVA 24 months following treatment with intravitreal aflibercept injection was 3.2 letters lower than that following 12 months in patients with CRVO [27]. Since CRVO is a retinal disease with a poor prognosis, long-term monitoring of the visual functions and QOL is important. Other factors known to affect stereopsis include pupil size [28], accommodation [5], and eye dominance [29]; however, these factors were not assessed in this study. Nevertheless, the influence of pupil size and eye dominance on stereopsis is negligible, and is unlikely to have affected our results. Even if the pupil size changed from $1 \mathrm{~mm}$ to $6 \mathrm{~mm}$, the change in TST score was 0.18 in a previous study [28], while the TST score changed by 0.2 with the change in the dominant eye [29]. Future studies with larger sample sizes and longer follow-up periods incorporating other factors are needed to further our understanding of stereopsis and visual function factors in patients with CRVO.

\section{Methods}

\section{Study design}

This multicenter, open-label, prospective study was conducted in accordance with the Declaration of Helsinki and with the approval of the Institutional Review Board of the University of Tsukuba Hospital and Mito Kyodo General Hospital. All the patients and normal subjects provided informed consent prior to inclusion in the study. Treatment-naïve patients with CRVO who were referred to the Tsukuba University Hospital or Mito Kyodo General Hospital were enrolled in this study. The inclusion criteria for participation 
were as follows: (1) center-involving macular edema secondary to CRVO, (2) central foveal thickness $(\mathrm{CFT})>250 \mu \mathrm{m}$, (3) patients aged 18 years or above, but younger than 85 years, and (4) patients who provided written informed consent. The exclusion criteria were as follows: (1) previous history of vitreoretinal surgery, (2) previous history of ophthalmic disorders, except mild refractive errors and mild cataract, (3) patients who underwent treatment for macular edema within the last 90 days (including subtenon triamcinolone acetonide, intravitreal bevacizumab, intravitreal ranibizumab [IVR], intravitreal aflibercept, topical steroid, and carbonic anhydrase inhibitors), (4) patients who underwent intraocular surgery within the past 90 days, (5) the contralateral eye of patients with CRVO, (6) patients with poorly controlled hypertension and diabetic mellitus, and (7) patients who underwent laser treatment within the last 30 days. We also included age-matched normal controls in this study.

\section{Study visits and assessments}

The stereopsis, best-corrected visual acuity (BCVA), and retinal microstructure were examined every month before treatment and over a period of 12 months after treatment. The VR-QOL was examined before treatment and at 3,6 , and 12 months following treatment. We converted the BCVA measured using the Landolt chart to the logarithm of the minimum angle of resolution (logMAR) for use in the subsequent analysis. The Titmus Stereo Test (TST) and the TNO stereotest (TNO) were performed under appropriate spectacle corrections with a standard viewing distance of $40 \mathrm{~cm}$ to evaluate the stereopsis. We flipped the stereo target and asked the patient if the target was in front or behind the page, and checked the response to ensure that the patient did not use monocular clues during the TST. The results for TST and TNO were expressed in "seconds of arc." These values were converted to logarithms for statistical evaluation [11].

The macular structure was evaluated using spectral-domain optical coherence tomography (OCT) (Cirrus high-definition OCT; Carl Zeiss, Dublin, CA). Five-line Raster Cross scanning was performed using the Cirrus analysis software version 3.0., and scans with a signal strength of more than $6 / 10$ were considered appropriate. The CFT was evaluated using the OCT image.

The VFQ-25 was administered to investigate the VR-QOL in patients with CRVO. The VFQ-25 consists of 25 items that permit patients to self-assess specific visual symptoms and difficulty with daily activities. The responses to the 25 questions are assigned to one of the following 12 subscales: general health, general vision, ocular pain, near activities, distance activities, social functioning, mental health, role limitations, dependency, driving, color vision, and peripheral vision. The composite VFQ-25 score is calculated as the average of the 11 subscale scores, excluding "general health." Subscales are scored on a scale of $0-100$ points, with 100 indicating the best possible functioning or minimal subjective impairment. This study utilized the Japanese version of the VFQ-25, which was modified to fit Japanese culture and lifestyle. The reliability and validity of the modified NEI VFQ-25 questionnaire have been assessed, and the questionnaire was proven to accurately measure the VR-QOL in Japanese individuals [30]. 


\section{Intraocular injections}

The patients with CRVO were administered three successive monthly injections of IVR ( 3 injections during months $0-2)$ ( $0.5 \mathrm{mg}$. Lucentis; Genetech) followed by pro re nata administration (3+PRN). After 3 injections (month 2), the participants were examined monthly and treated with intravitreal injections on a PRN basis according to the retreatment protocol. The criteria for PRN re-injection were as follows: (1) CFT $\geq 300 \mu \mathrm{m}$ in the study eye as assessed by OCT, (2) detection of new cystoid changes in the retina, retinal bleeding or subretinal fluid on OCT, and (3) a decrease in visual acuity $>0.1$ logMAR compared to the values obtained at the last visit.

The injection protocol was as follows: an eyelid opener was applied following topical anesthesia instillation and the injection site was washed with povidone iodine. A 30-gauge needle was inserted through the pars plana and $0.05 \mathrm{~mL}$ of ranibizumab was injected. All the procedures were performed at our clinic by experienced vitreoretinal surgeons (T.M., Y.S., and S.M.).

\section{Statistical analysis}

The mean and standard deviation values were calculated for each parameter. The

unpaired t-test was used to compare the age, stereopsis, and BCVA between patients with CRVO and normal controls. The chi-squared test was performed to determine the presence of any sex-based between-group differences. The Wilcoxon signed-rank test was performed to investigate changes in the visual function (TST, TNO, and BCVA), CFT, and composite VFQ-25 score. The associations between stereopsis and the composite VFQ-25 score and BCVA were examined using the Spearman rankcorrelation test. Multivariate analysis was performed to investigate the relationship between the VR-QOL and visual functions. All the analyses were conducted using SPSS (version 27, IBC Corp., Chicago, IL, USA). $P$-values less than 0.05 were considered statistically significant.

\section{Declarations}

Acknowledgements: None

Financial Support: This study was supported by Novartis Pharma K.K., Tokyo, Japan. No additional external funding was received for this study. The sponsor had no role in the design or conduct of this research.

Competing Interests: This study was supported by Novartis Pharma K.K., Tokyo, Japan. No additional external funding was received for this study. The sponsor had no role in the design or conduct of this research.

\section{Author contributions:}

Fumiki Okamoto and Tetsuro Oshika conduct this study. 
Fumiki Okamoto wrote the main manuscript text.

Shohei Morikawa, Yoshimi Sugiura, and Tomoya Murakami had data collection.

Mizuki Tomioka and Takahiro Hiraoka had data analysis.

All authors reviewed the manuscript.

\section{References}

1. Lam, A.K., Chau, A. S., Lam, W. Y., Leung, G. Y. \& Man, B. S. Effect of naturally occurring visual acuity differences between two eyes in stereoacuity. Ophthalmic Physiol. Opt. 16, 189-195 (1996).

2. Donzis, P.B., Rappazzo, J. A., Burde, R. M. \& Gordon, M. Effect of binocular variations of Snellen's visual acuity on Titmus stereoacuity. Arch. Ophthalmol. 101, 930-932 (1983).

3. Larson, W.L. \& Bolduc, M. Effect of induced blur on visual acuity and stereoacuity. Optom. Vis. Sci. 68, 294-298 (1991).

4. Levy, N.S. \& Glick, E.B. Stereoscopic perception and Snellen visual acuity. Am. J. Ophthalmol. 78, 722-724 (1974).

5. Goodwin, R.T. \& Romano, P.E. Stereoacuity degradation by experimental and real monocular and binocular amblyopia. Invest. Ophthalmol. Vis. Sci. 26, 917-923 (1985).

6. Watanabe H., et al. Stereopsis after successful surgery for rhegmatogenous retinal detachment. Graefes Arch. Clin. Exp. Ophthalmol. 252, 1207-1212 (2014).

7. Lina, G., Xuemin, Q., Qinmei, W. \& Lijun, S. Vision-related quality of life, metamorphopsia, and stereopsis after successful surgery for rhegmatogenous retinal detachment. Eye (Lond) 30,40-45 (2016).

8. Okamoto, F., et al. Preoperative aniseikonia is a prognostic factor for postoperative stereopsis in patients with unilateral epiretinal membrane. Graefes Arch. Clin. Exp. Ophthalmol. 258, 743-749 (2020).

9. Okamoto, F., Sugiura, Y., Okamoto, Y., Hiraoka, T. \& Oshika, T. Stereopsis and optical coherence tomography findings after epiretinal membrane surgery. Retina 35, 1415-1421 (2015).

10. Okamoto, F., et al. Vision-related parameters that affect stereopsis in patients with macular hole. Sci. Rep. 10, 2805 (2020).

11. Okamoto, F., et al. Stereopsis and retinal microstructures following macular hole surgery. Sci. Rep. 10, 19534 (2020).

12. Morikawa, S., et al. Stereopsis after intravitreal ranibizumab injections for branch retinal vein occlusion. Ophthalmol. Retina 3, 777-783 (2019).

13. Mangione, C.M., et al; National Eye Institute Visual Function Questionnaire Field Test Investigators. Development of the 25-item National Eye Institute Visual Function Questionnaire. Arch. Ophthalmol. $119,1050-1058$ (2001). 
14. Campochiaro, P.A., et al. Ranibizumab for macular edema following branch retinal vein occlusion: six-month primary end point results of a phase III study. Ophthalmology 117, 1102-1112 (2010).

15. Clark, W.L., et al. Intravitreal aflibercept for macular edema following branch retinal vein occlusion: 52-week results of the VIBRANT study. Ophthalmology 123, 330-336 (2016).

16. Okamoto, F., Okamoto, Y., Hiraoka, T. \& Oshika, T. Effect of vitrectomy for epiretinal membrane on visual function and vision-related quality of life. Am. J. Ophthalmol. 147, 869-874 (2009).

17. Okamoto, F., Okamoto, Y., Hiraoka, T. \& Oshika, T. Vision-related quality of life and visual function after retinal detachment surgery. Am. J. Ophthalmol. 146, 85-90 (2008).

18. Ng, H., Vermeer, K.A., van Meurs, J.C. \& La Heij, E.C. Visual acuity inadequately reflects vision-related quality of life in patients after macula-off retinal detachment surgery. Invest. Ophthalmol. Vis. Sci. 61, 34 (2020).

19. Potic, J., et al. Application of modified NEI VFQ-25 after retinal detachment to vision-related quality of life. Retina. 10.1097/IAE.0000000000002894 (2020).

20. Fukuda, S., et al. Vision-related quality of life and visual function in patients undergoing vitrectomy, gas tamponade and cataract surgery for macular hole. Br. J. Ophthalmol. 93, 1595-1599 (2009).

21. Okamoto, F., Okamoto, Y., Fukuda, S., Hiraoka, T. \& Oshika, T. Vision-related quality of life and visual function after vitrectomy for various vitreoretinal disorders. Invest. Ophthalmol. Vis. Sci. 51, 744-751 (2010).

22. Campochiaro, P.A., et al. Sustained benefits from ranibizumab for macular edema following central retinal vein occlusion: twelve-month outcomes of a phase III study. Ophthalmology 118, 2041-2049 (2011).

23. Korobelnik, J.F., et al; GALILEO Study Group. Intravitreal aflibercept injection for macular edema resulting from central retinal vein occlusion: One-year results of the phase 3 GALILEO study. Ophthalmology 121, 202-208 (2014).

24. Mamou, J., et al. Ultrasound-based quantification of vitreous floaters correlates with contrast sensitivity and quality of life. Invest Ophthalmol. Vis. Sci. 56, 1611-1617 (2015).

25. Matza, L.S., Rousculp, M. D., Malley, K., Boye, K. S. \& Oglesby, A. The longitudinal link between visual acuity and health-related quality of life in patients with diabetic retinopathy. Health Qual. Life Outcomes 6, 95 (2008).

26. Frick, K.D., et al; Multicenter Uveitis Steroid Treatment-MUST Trial Research Group. Associations among visual acuity and vision- and health-related quality of life among patients in the multicenter uveitis steroid treatment trial. Invest. Ophthalmol. Vis. Sci. 53, 1169-1176 (2012).

27. Heier, J.S., et al. Intravitreal aflibercept injection for macular edema due to central retinal vein occlusion: two-year results from the COPERNICUS study. Ophthalmology 121, 1414-1420 (2014).

28. Lovasik, J.V. \& Szymkiw, M. Effects of aniseikonia, anisometropia, accommodation, retinal illuminance, and pupil size on stereopsis. Invest. Ophthalmol. Vis. Sci. 26, 741-750 (1985). 
29. Erickson, P. \& McGill, E.C. Role of visual acuity, stereoacuity, and ocular dominance in monovision patient success. Optom. Vis. Sci. 69, 761-764 (1992).

30. Suzukamo, Y., et al. Psychometric properties of the 25-item National Eye Institute Visual Function Questionnaire (NEI VFQ-25), Japanese version. Health Qual. Life Outcomes 3, 65 (2005).

\section{Tables}

Table 1. Baseline characteristics of patients with central retinal vein occlusion (CRVO) and normal controls

\begin{tabular}{|llll|}
\hline & CRVO & Normal controls & $p$ values \\
\hline Age (years) & $72.2 \pm 11.1$ & $68.0 \pm 6.4$ & $\mathrm{p}=0.25$ \\
\hline Sex (men/women) & $13 / 10$ & $6 / 7$ & $\mathrm{p}=0.55$ \\
\hline Titmus Stereo Test (log) & $3.17 \pm 0.70$ & $1.72 \pm 0.19$ & $\mathrm{p}<0.001$ * \\
\hline TNO stereotest (log) & $3.27 \pm 0.59$ & $1.85 \pm 0.23$ & $\mathrm{p}<0.001$ * \\
\hline BCVA (logMAR) & $0.79 \pm 0.56$ & $-0.07 \pm 0.07$ & $\mathrm{p}<0.001$ * \\
\hline Central foveal thickness $(\mu \mathrm{m})$ & $770 \pm 319$ & - & - \\
\hline VFQ-25 composite score (point) & $62.6 \pm 16.9$ & - & - \\
\hline Duration of disease (months) & $2.1 \pm 2.5$ & - & - \\
\hline
\end{tabular}

Values are presented as the mean \pm standard deviation.

$\mathrm{BCVA}=$ best-corrected visual acuity, logMAR $=$ logarithm of the minimum angle of resolution, VFQ-25 = 25-item National Eye Institute Visual Function Questionnaire

Table 2. Correlation between the composite VFQ-25 score and visual functions in central retinal vein occlusion 


\begin{tabular}{|c|c|c|}
\hline & \multicolumn{2}{|c|}{ Composite VFQ-25 score at baseline } \\
\hline & $\mathrm{r}$ & $\mathrm{p}$ \\
\hline Titmus Stereo Test at baseline & -0.450 & 0.036 * \\
\hline TNO stereotest at baseline & -0.419 & 0.052 \\
\hline \multirow[t]{4}{*}{ BCVA at baseline } & -0.184 & 0.412 \\
\hline & \multicolumn{2}{|c|}{ Composite VFQ-25 score } \\
\hline & \multicolumn{2}{|c|}{12 months post-treatment } \\
\hline & $r$ & $\mathrm{P}$ \\
\hline Titmus Stereo Test at 12 months post-treatment & -0.419 & 0.094 \\
\hline TNO stereotest at 12 months post-treatment & -0.660 & 0.004 * \\
\hline \multirow[t]{4}{*}{ BCVA at 12 months post-treatment } & -0.554 & 0.021 * \\
\hline & \multicolumn{2}{|c|}{ Composite VFQ-25 score } \\
\hline & \multicolumn{2}{|c|}{12 months post-treatment } \\
\hline & $r$ & $\mathrm{P}$ \\
\hline Titmus Stereo Test at baseline & -0.482 & 0.043 * \\
\hline TNO stereotest at baseline & -0.595 & 0.009 * \\
\hline BCVA at baseline & -0.553 & 0.017 * \\
\hline
\end{tabular}

BCVA = best-corrected visual acuity, VFQ-25 = 25-item National Eye Institute Visual Function Questionnaire

\section{Figures}



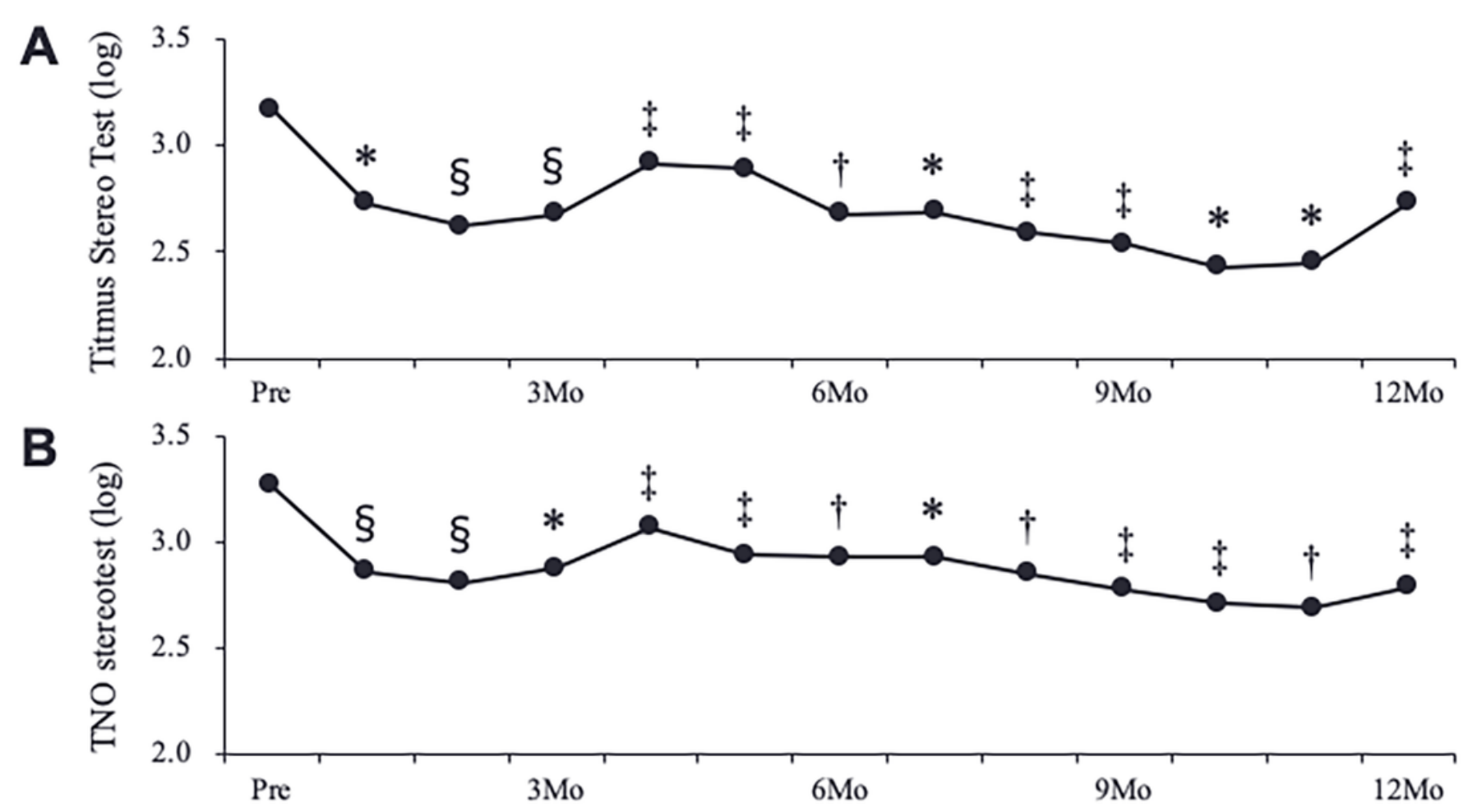

\section{Figure 1}

Changes in the Titmus Stereo Test (A) and TNO stereotest (B) pre- and post-treatment in patients with central retinal vein occlusion $\S p<0.001,{ }^{\star} p<0.005,+p<0.01, \neq p<0.05$ compared to baseline

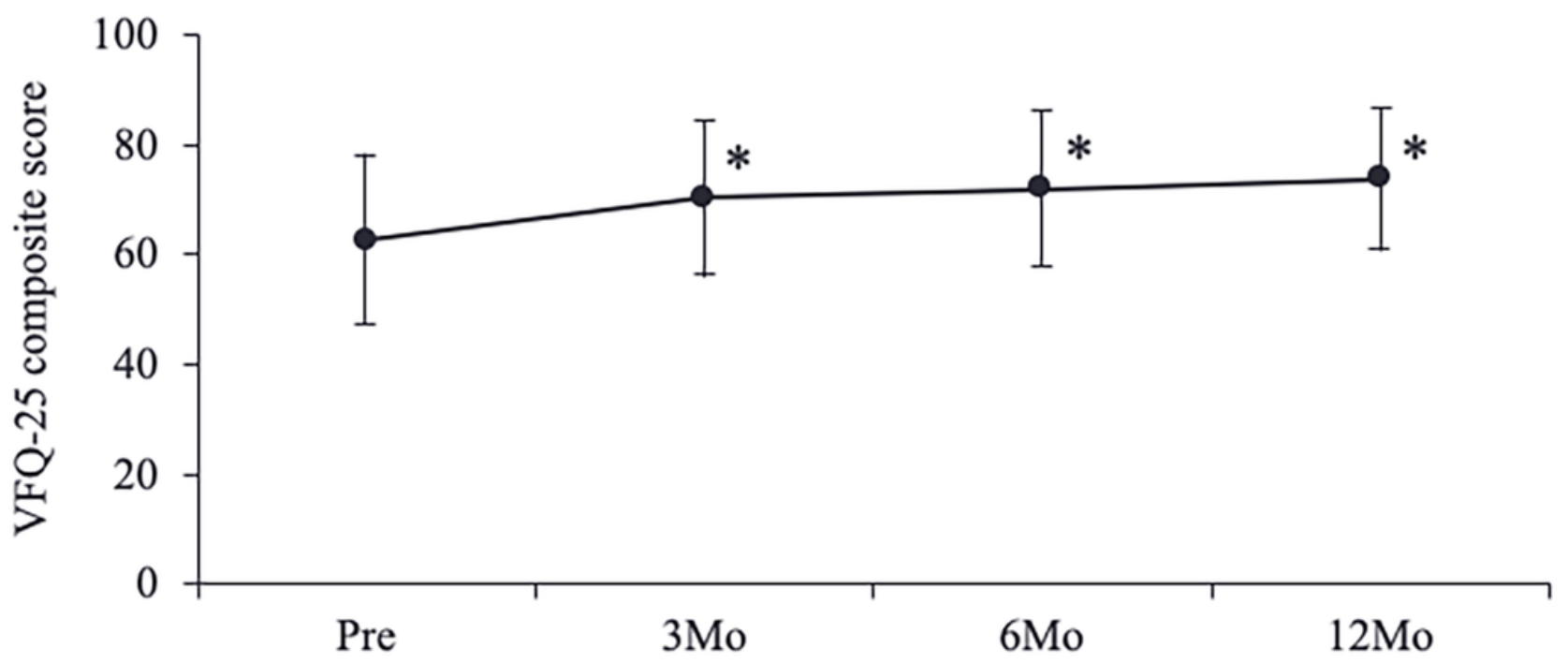


Figure 2

Changes in the composite VFQ-25 score pre- and post-treatment in patients with central retinal vein occlusion * $\mathrm{p}<0.005$ compared to baseline The error bars indicate standard deviations. VFQ-25 $=25$-Item National Eye Institute Visual Function Questionnaire

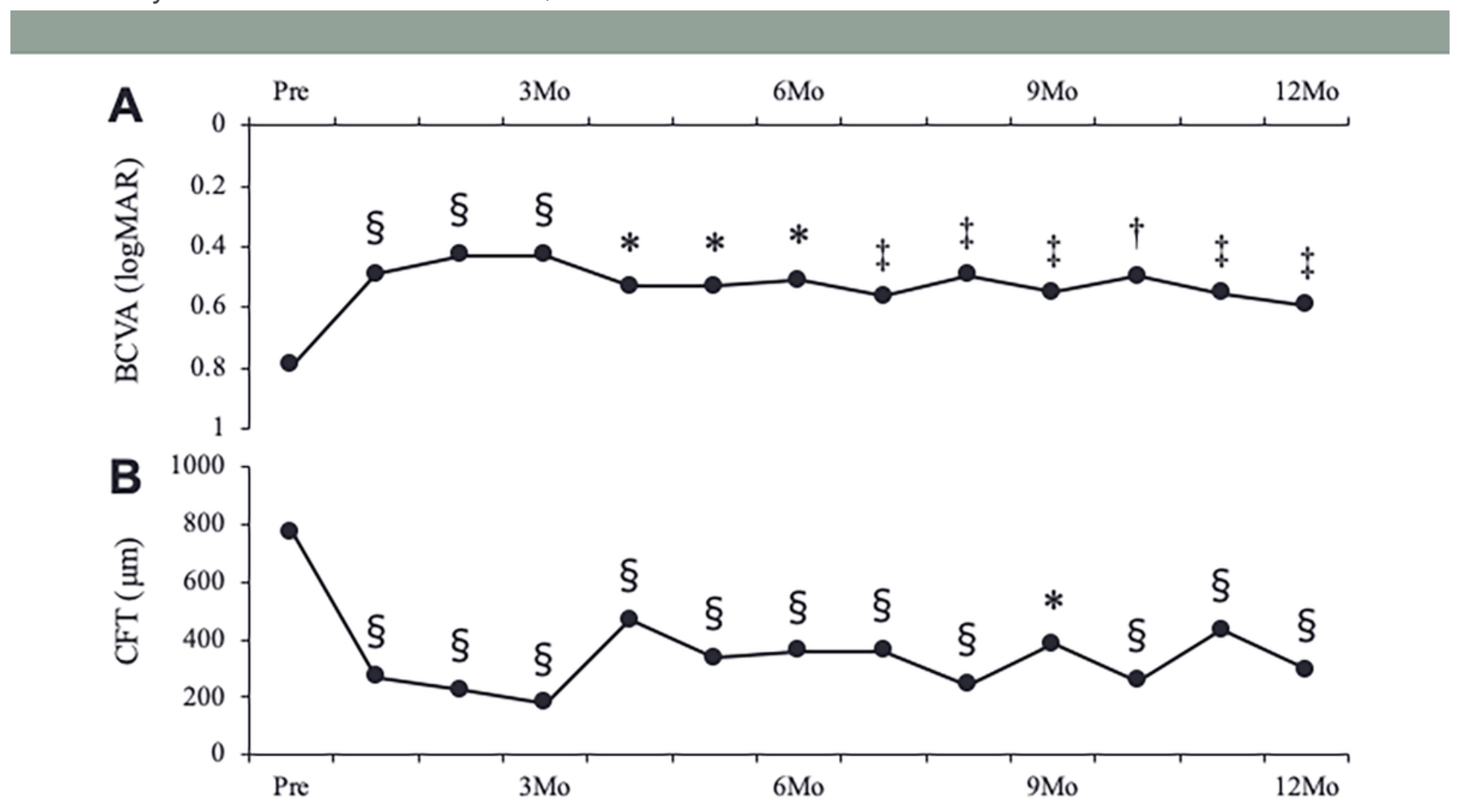

Figure 3

Changes in the BCVA (A) and CFT (B) pre-and post-treatment in patients with central retinal vein occlusion $\S p<0.001,{ }^{\star} p<0.005,+p<0.01, \neq p<0.05$ compared to baseline $B C V A=$ best-corrected visual acuity, CFT = central foveal thickness 

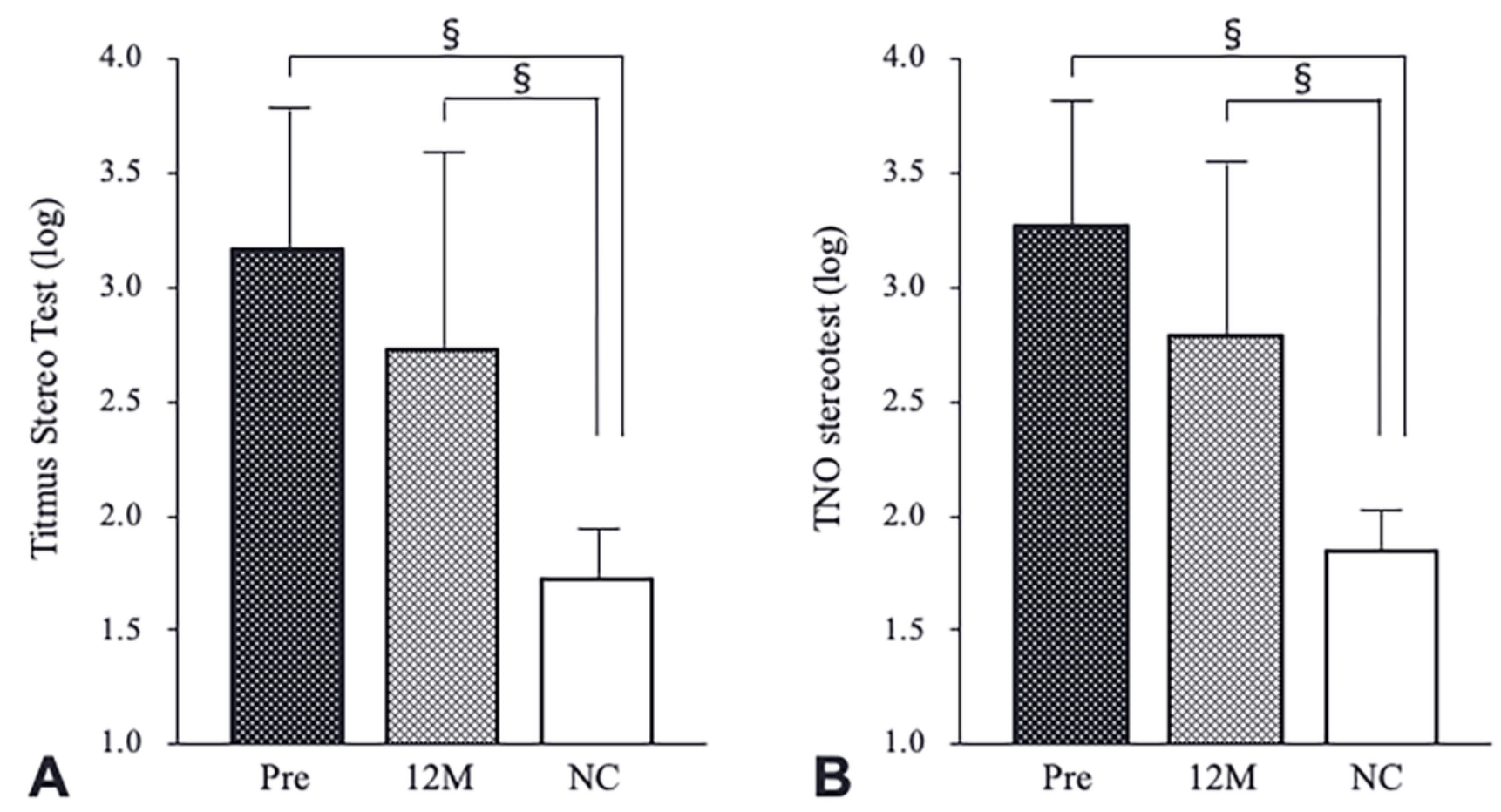

Figure 4

A. Titmus Stereo Test values of patients with central retinal vein occlusion pre- and post-treatment 12 months and those of the normal controls (NCs) B. TNO stereotest values of patients with central retinal vein occlusion pre- and post-treatment 12 months and those of the normal controls (NCs) The error bars indicate standard deviations. $\S p<0.001$ LogMAR: logarithm of the minimum angle of resolution 